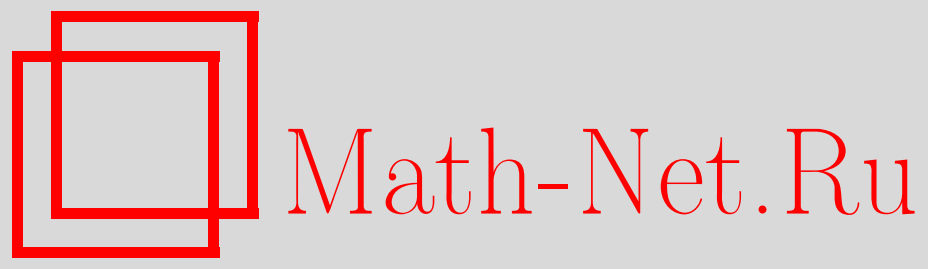

Г. В. Розенблюм, М. З. Соломяк, О спектральных оценках для операторов типа Шрёдингера: случай малой локальной размерности, Функи. анализ и его прил., 2010, том 44, выпуск 4, 21-33

DOI: https://doi.org/10.4213/faa3018

Использование Общероссийского математического портала Math$\mathrm{Net.Ru}$ подразумевает, что вы прочитали и согласны с пользовательским соглашением

http://www. mathnet.ru/rus/agreement

Параметры загрузки:

IP : 54.198 .67 .100

26 апреля 2023 г., 14:17:26

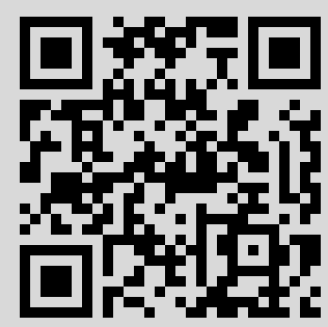


Функционалъный анализ и его приложения

2010, т. 44, вып. 4, с. 21-33

УДК $517.983+517.93$

\title{
О спектральных оценках для операторов типа Шрёдингера: случай малой локальной размерности
}

\author{
(C) 2010. Г. В. РозеньлЮм, М. З. Соломяк
}

Светлой памяти М. Ш. Бирмана, Ученого и Человека

\begin{abstract}
Поведение дискретного спектра оператора Шрёдингера $-\Delta-V$ в значительной степени определяется поведением соответствующего ядра теплопроводности $P(t ; x, y)$ при $t \rightarrow 0$ и $t \rightarrow \infty$. В случае его степенных оценок

$$
\|P(t ; \cdot, \cdot)\|_{L^{\infty}}=O\left(t^{-\delta / 2}\right), \quad t \rightarrow 0, \quad\|P(t ; \cdot, \cdot)\|_{L^{\infty}}=O\left(t^{-D / 2}\right), \quad t \rightarrow \infty,
$$

естественно называть показатели $\delta, D$ локалъной размерностъю и размерностью на бесконечности соответственно. Характер спектральных оценок зависит от соотношения между этими размерностями. Мы рассматриваем случай $\delta<D$, ранее изученный недостаточно. В качестве приложений рассматриваются операторы на комбинаторных и метрических графах.
\end{abstract}

\section{§1. Введение}

Среди работ М. Ш. Бирмана едва ли не наибольшее влияние на развитие спектральной теории оказала статья [2] (1961). Разработанный в ней подход, получивший впоследствии название «принцип Бирмана-Швингера», стал источником вдохновения и одним из главных инструментов в спектральном анализе операторов типа Шрёдингера.

В [13] этот подход был применен для получения оценок собственных значений таких операторов в весьма общей ситуации, и выяснилось, что эти оценки существенно зависят от двух численных характеристик оператора, $\delta$ и $D$, которые могут быть названы локальной размерностью и размерностью на бесконечности. Для обычного оператора Шрёдингера в $\mathbb{R}^{d}$ эти характеристики совпадают с размерностью; в общей ситуации $\delta \neq D$.

В обзоре [14] обсуждались различные соотношения между размерностями, причем основное внимание было уделено эффектам, возникающим при $\delta \geqslant D$. Простейший пример с $\delta<D$ дается дискретным оператором Шрёдингера на решетке $\mathbb{Z}^{d}$; здесь $D=d$ и $\delta=0$. В этом случае некоторые особенности в распределении спектра были обнаружены в [15]. В частности, оценки собственных значений при большой константе связи, точные по порядку в $\mathbb{R}^{d}$, в случае $\mathbb{Z}^{d}$ уже перестают быть точными. Более глубокое изучение явлений, обнаруженных в [15], привело авторов к пониманию того, что такие свойства типичны для общей ситуации $\delta<D$. В настоящей статье мы рассматриваем случаи достаточно общих комбинаторных графов, для которых $\delta=0$, и квантовых (метрических) графов, для которых $\delta>1$ сколь угодно близко к 1 ; мы ограничиваемся рассмотрением случая $D>2$. Мы находим серию оценок спектра для операторов Шрёдингера на таких графах. Оказывается, что для квантовых графов такие 
оценки определяются оценками для ассоциированных комбинаторных графов, что довольно неожиданно, поскольку квантовый граф гораздо богаче как множество. Это явление отчасти объясняется теоремой 4.1, в которой установлены соотношения между размерностями этих графов. Мы также устанавливаем критерии принадлежности оператора Бирмана-Швингера классам Шаттена и «слабым» классам Шаттена и условия, при которых имеют место вейлевские асимптотики собственных значений. Главные результаты в этих направлениях - теоремы 5.3 и 5.5 .

Первый автор выражает благодарность Институту Вейцманна за гостеприимство и поддержку. Авторы благодарны Т. А. Суслиной и рецензентам за внимательное чтение рукописи и полезные советы.

\section{§2. Операторы на общих пространствах с мерой}

2.1. Размерности полугруппы. Пусть $(X, \sigma)$ - пространство с сигмаконечной мерой. Положим $L^{q}(X)=L^{q}(X, \sigma)$ и $\|\cdot\|_{q}=\|\cdot\|_{L^{q}(X)} \cdot$ Далее мы часто опускаем указание на пространство $X$. Пусть $\mathbf{A}$ - неотрицательный самосопряженный оператор в $L^{2}(X)$ и $\mathbf{P}(t)=\exp (-\mathbf{A} t)-$ соответствующая полугруппа. Мы предполагаем, что для всех $t>0$ оператор $\mathbf{P}(t)$ сохраняет положительность и ограничен как оператор из $L^{1}$ в $L^{\infty}$. Хорошо известно, что при этих предположениях $\mathbf{P}(t)$ - интегральный оператор, ядро $P(t ; x, y)$ которого (ядро теплопроводности) корректно определено для $t>0$ как функция из $L^{\infty}(X \times X)$ (детали см. в [13]). Положим

$$
M_{\mathbf{A}}(t)=\|P(t ; \cdot, \cdot)\|_{L^{\infty}(X \times X)} \cdot
$$

Описанный выше класс операторов $\mathbf{A}$ (будем обозначать его через $\mathscr{P}$ ) включает непрерывный и дискретный лапласианы и много других важных операторов; см. [13], [10]. Для простоты любой оператор $\mathbf{A} \in \mathscr{P}$ мы будем называть лапласианом.

Функция $M_{\mathbf{A}}(t)$ невозрастающая. Главные ее характеристики - поведение при $t \rightarrow 0$ и $t \rightarrow \infty$. Всюду в этой статье мы предполагаем, что найдутся два неотрицательных показателя $\delta, D$, таких, что

$$
M_{\mathbf{A}}(t)=O\left(t^{-\delta / 2}\right), \quad t \rightarrow 0, \quad M_{\mathbf{A}}(t)=O\left(t^{-D / 2}\right), \quad t \rightarrow \infty,
$$

и, более того, что

$$
D \geqslant \delta, \quad D>2 \text {. }
$$

Обозначим через а квадратичную форму оператора А. Из (2.1), (2.2) следует, что для некоторой константы $C$ при всех $t \in(0,+\infty)$ имеет место оценка $M_{\mathbf{A}}(t) \leqslant C t^{-D / 2}$. По теории Варопулоса [17] последнее неравенство равносильно «теореме вложения»

$$
\|u\|_{L^{p}}^{2} \leqslant C \mathbf{a}[u], \quad p=p(D)=2 D /(D-2) \quad \forall u \in \operatorname{Dom}(\mathbf{a}) .
$$

Обозначим через $H_{\mathbf{a}}$ пополнение области определения формы а относительно а-нормы $\|u\|_{\mathbf{a}}=\sqrt{\mathbf{a}[u]}$. В силу $(2.3) H_{\mathbf{a}}$ может быть реализовано как пространство функций на $X$ (вложенное в $L^{p}$ ).

2.2. Общие оценки собственных значений. Пусть $V \geqslant 0$ - измеримая функция на $X$. При некоторых дополнительных предположениях оператор 
$\mathbf{A}-V$, определенный через квадратичную форму $\mathbf{a}[u]-\int_{X} V|u|^{2} d \sigma$, является самосопряженным, причем его отрицательный спектр состоит из конечного числа собственных значений конечной кратности. Ниже $N_{-}(\mathbf{A}-V)$ обозначает суммарную кратность отрицательного спектра оператора $\mathbf{A}-V$. В [13] доказано, что число $N_{-}(\mathbf{A}-V)$ допускает оценку в терминах функции $M_{\mathbf{A}}(t)$. Вывод этой оценки является абстрактной версией подхода Либа к доказательству оценки Розенблюма-Либа-Цвикеля.

Оценки величины $N_{-}(\mathbf{A}-V)$ зависят от того, какой из показателей в $(2.1), \delta$ или $D$, больше. В частности, имеет место следующий вариант оценки Розенблюма-Либа-Цвикеля (детали доказательства см. в [14] (замечание 1 в разд. 3.2), а также в [13], особенно разд. 3.1 с $d$, замененным на $D)$. Мы формулируем результат для оператора с большим параметром $\alpha>0$ (константой связи).

Теорема 2.1. Предположим, что $D \geqslant \delta, D>2$. Тогда для всякой функиии $V \in L^{D / 2}(X)$ и любого $\alpha>0$ оператор $\mathbf{A}-\alpha V$ корректно определен, его отрицательный спектр конечен и

$$
N_{-}(\mathbf{A}-\alpha V) \leqslant C \alpha^{D / 2} \int_{X} V^{D / 2} d \sigma, \quad C=C(X, D) .
$$

Оценки этого типа удобно формулировать в терминах соответствующего оператора Бирмана-Швингера $\mathbf{B}_{V}$. Напомним (см., например, [2], [5]), что $\mathbf{B}_{V}$ определяется как оператор в $H_{\mathbf{a}}$, порожденный квадратичной формой

$$
\mathbf{b}_{V}[u]=\int_{X} V|u|^{2} d \sigma .
$$

Благодаря неравенству (2.3) при $V \in L^{D / 2}$ этот оператор корректно определен. Отношение Рэлея для оператора $\mathbf{B}_{V}$ равно $\mathbf{b}_{V}[u] / \mathbf{a}[u], u \in H_{\mathbf{a}}$; функция распределения его собственных значений обозначается через $n\left(s, \mathbf{B}_{V}\right)$.

Теорема 2.1 может быть переформулирована следующим образом:

Теорема 2.2. B предположениях теоремы $2.1 \mathbf{B}_{V} \in \Sigma_{D / 2} u$

$$
\left\|\mathbf{B}_{V}\right\|_{\Sigma_{D / 2}} \leqslant C\|V\|_{L^{D / 2}} .
$$

Напомним, что $\Sigma_{p}$ обозначает класс компактных операторов со степенной оценкой сингулярных чисел, $s_{n}(T)=O\left(n^{-1 / p}\right)$, см. [4, разд. 11.6]. Аналогичные классы с о вместо $O$ обозначаются через $\Sigma_{p}^{(0)}$; они замкнуты в $\Sigma_{p}$. Ниже мы также используем стандартные классы фон Неймана-Шаттена $\mathfrak{S}_{p}, 0<p \leqslant \infty$.

Пусть теперь $\delta<D$. Тогда из (2.1) следует, что $M_{\mathbf{A}}(t)=O\left(t^{-q}\right)$ при $t \rightarrow 0, \infty$ с любым $q \in[\delta / 2, D / 2]$. Отсюда получаем оценку, аналогичную $(2.6)$, с любым таким показателем $q$ вместо $D / 2$ (и константой $C$, зависящей от $q$ ):

$$
\left\|\mathbf{B}_{V}\right\|_{\Sigma_{q}} \leqslant C\|V\|_{L^{q}}, \quad \delta / 2 \leqslant q \leqslant D / 2, q>1
$$

Известно, что в случае лапласиана в $\mathbb{R}^{d}, d \geqslant 3$ (тогда $D=\delta=d$ ), оценка (2.6) точна в том смысле, что при $V \not \equiv 0$ оператор $\mathbf{B}_{V}$ не может принадлежать никакому шаттеновскому идеалу, меньшему, чем $\Sigma_{d / 2}$. В [15] было показано, что для лапласиана на решетке ситуация иная: если $V \in \ell^{d / 2}\left(\mathbb{Z}^{d}\right), d \geqslant 3$, то $\mathbf{B}_{V} \in$ $\Sigma_{d / 2}^{(0)}\left(\right.$ или, другими словами, $\left.N_{-}(\mathbf{A}-\alpha V)=o\left(\alpha^{d / 2}\right)\right)$. Наш следующий результат показывает, что похожий факт имеет место в общем случае, если $D>\delta$. 
Теорема 2.3. Пусть выполнены предположения теоремы 2.1, и пусть $D>\delta$. Тогда $\mathbf{B}_{V} \in \Sigma_{D / 2}^{(0)}$.

Доказательство. Фиксируем число $q \in(\max (\delta / 2,1), D / 2)$. Функции $V \in$ $L^{D / 2} \cap L^{\delta / 2}$ лежат в $L^{q}$ и плотны в $L^{D / 2}$. Для таких функций $V$ из оценки $(2.7)$ получаем, что $\mathbf{B}_{V} \in \Sigma_{q} \subset \Sigma_{D / 2}^{(0)}$. По непрерывности (см. [4, теорема 11.6.7]) это включение имеет место для всех $V \in L^{D / 2}$.

Следующий результат показывает, что оценка (2.7) с произвольным $q<D / 2$ не точна в том смысле, что класс допустимых потенциалов можно существенно расширить и оператор $\mathbf{B}_{V}$ для $V \in L^{q}$ в действительности лежит в меньшем, чем $\Sigma_{q}$, классе $\mathfrak{S}_{q}$. Обозначим через $L_{w}^{q}$ слабое $L^{q}$-пространство (см., например, [1]).

Теорема 2.4. Пусть выполнены предположения теоремы 2.1 , причем $D>\delta$. Тогда для любого $q \in(\max (\delta / 2,1), D / 2)$

$$
V \in L_{w}^{q} \Longrightarrow \mathbf{B}_{V} \in \Sigma_{q}, \quad V \in L^{q} \Longrightarrow \mathbf{B}_{V} \in \mathfrak{S}_{q}
$$

с оценками

$$
\left\|\mathbf{B}_{V}\right\|_{\Sigma_{q}} \leqslant C\|V\|_{L_{w}^{q}}, \quad\left\|\mathbf{B}_{V}\right\|_{\mathfrak{S}_{q}} \leqslant C\|V\|_{L^{q}} .
$$

Иначе говоря,

$$
N_{-}(\mathbf{A}-\alpha V) \leqslant C \alpha^{q}\|V\|_{L_{w}^{q}}^{q}
$$

$u N_{-}(\mathbf{A}-\alpha V)=o\left(\alpha^{q}\right), \alpha \rightarrow \infty, \partial л я V \in L^{q}$.

Этот результат получается из (2.7) с помощью вещественной интерполяции (cM. [1]).

\section{§3. Комбинаторные графы}

Оставшаяся часть статьи посвящена операторам на графах. В этом параграфе мы рассматриваем комбинаторные графы (которые обозначаем через $G$ ), в двух следующих - метрические (обозначаем через $Г$ ). В обоих случаях мы всегда предполагаем, что граф связный, имеет бесконечно много вершин и не имеет петель, висячих вершин и кратных ребер (основные понятия теории графов см., например, в [9]). Обозначим через $\mathscr{E}$ и $\mathscr{V}$ множества ребер и вершин графа соответственно. Если вершины $v$ и $v^{\prime}$ смежны (соединены ребром $e$ ), будем писать $v \sim v^{\prime}$ или иногда $e=\left(v, v^{\prime}\right)$. Мы всегда предполагаем, что степени всех вершин графа конечны:

$$
\operatorname{deg}(v)=\#\left\{v^{\prime} \in \mathscr{V}, v^{\prime} \sim v\right\}<\infty \quad \forall v \in \mathscr{V} .
$$

Каждому ребру графа $e \in \mathscr{E}$ поставим в соответствие вес $g_{e}>0$. Рассмотрение таких графов с весами вызвано потребностями $\S \S 4,5$.

На множестве $\mathscr{V}$ рассмотрим считающую меру $\sigma, \sigma(v)=1$ для любой вершины $v \in \mathscr{V}$. В этом параграфе основное гильбертово пространство - это $\ell^{2}(\mathscr{V})=$ $L^{2}(\mathscr{V}, \sigma)$; также будем использовать обозначения $\ell^{q}=L^{q}(\mathscr{V}, \sigma), \ell_{w}^{q}=L_{w}^{q}(\mathscr{V}, \sigma)$. Квадратичная форма

$$
\mathbf{a}_{G}[f]=\sum_{e \in \mathscr{E} ; e=\left(v, v^{\prime}\right)} g_{e}\left|f(v)-f\left(v^{\prime}\right)\right|^{2}
$$


с областью определения $\left\{f \in \ell^{2}(\mathscr{V}), \mathbf{a}_{G}[f]<\infty\right\}$ задает в $\ell^{2}(\mathscr{V})$ неотрицательный самосопряженный оператор $\mathbf{A}=\mathbf{A}_{G}=-\Delta_{G}$. В частности, если веса $g_{e}$ и степени $\operatorname{deg}(v)$ равномерно ограничены, то оператор $\mathbf{A}$ ограничен. В силу включения $\ell^{1}(\mathscr{V}) \subset \ell^{2}(\mathscr{V}) \subset \ell^{\infty}(\mathscr{V})$ операторы $\exp (-\mathbf{A} t)$ ограничены как операторы из $\ell^{1}$ в $\ell^{\infty}$, так что $M_{\mathbf{A}}(t) \leqslant C$. Это означает, что $\delta=0$. Наше главное предположение (ср. (2.1), (2.2)) - справедливость оценки

$$
M_{\mathbf{A}_{G}}(t)=O\left(t^{-D / 2}\right) \quad \text { с некоторым } D>2, t \rightarrow \infty .
$$

Соответствующее неравенство вида (2.3)

$$
\|f\|_{\ell^{p}}^{2} \leqslant C \mathbf{a}_{G}[f], \quad p=p(D)=2 D(D-2)^{-1},
$$

очевидным образом выполняется для всех $f$ с конечным носителем. Следовательно, замыкание $\mathscr{H}(G)$ множества таких функций по метрике $\mathbf{a}_{G}[f]$ вкладывается в $\ell^{p}$. В обозначениях $\S 2$ роль пространства $H_{\mathbf{a}}$ играет $\mathscr{H}(G)$. Линейное подпространство в $\mathscr{H}(G)$ функций, имеющих конечный носитель, будем обозначать через $\mathscr{H}_{\text {fin }}(G)$.

Имеется много геометрических и аналитических критериев выполнения соотношения (3.2). Не вдаваясь в детали, мы отсылаем читателя к [6], [8], [16], где приведены такие критерии. Пример графа, удовлетворяющего (3.2), - целочисленная решетка $\mathbb{Z}^{d}$; для каждого ребра e мы полагаем $g_{e}=1$. Здесь $D=d$, что можно проверить прямым вычислением ядра теплопроводности. Этот оператор изучался нами в работе [15]. Здесь мы распространяем некоторые из ее результатов на случай общих графов.

Следующий результат во многом является лишь частным случаем теорем 2.2-2.4 ${ }^{1}$. Единственное новшество - снятие неявного условия $q>1$ теоремы 2.4. Заметим, что в нашей ситуации $\mathbf{b}_{V}$ принимает вид

$$
\mathbf{b}_{V}[f]=\sum_{v \in \mathscr{V}} V(v)|f(v)|^{2} .
$$

Теорема 3.1. Пусть справедлива оценка (3.2).

1. Предположим, что $V \in \ell^{D / 2}(\mathscr{V})$. Тогда

$$
\left\|\mathbf{B}_{V}\right\|_{\Sigma_{D / 2}} \leqslant C\|V\|_{\ell^{D / 2}}
$$

при этом $\mathbf{B}_{V} \in \Sigma_{D / 2}^{(0)}$

2. Если $V \in \ell_{w}^{q}(G)$ (или $\left.V \in \ell^{q}(G)\right)$ для некоторого $q \in(0, D / 2)$, то $\mathbf{B}_{V} \in \Sigma_{q}$ (соответственно $\mathbf{B}_{V} \in \mathfrak{S}_{q}$ ) и справедливы оценки (2.8).

Доказательство (такое же, как в случае $G=\mathbb{Z}^{d}$, см. [15]) опускается.

В отличие от общей ситуации $\S 2$, для графов возможно получить также нижние оценки величины $n\left(s, \mathbf{B}_{V}\right)$ в терминах функции распределения для $V$, т. е.

$$
\nu(\tau, V)=\# E(\tau, V), \quad E(\tau, V)=\{v \in \mathscr{V}: V(v)>\tau\}, \quad \tau>0 .
$$

В этой оценке на $V$ не налагается никаких ограничений. Нам, однако, понадобятся два дополнительных предположения о графе $G$ : веса и степени вершин

1) Здесь и далее мы формулируем результаты только в терминах операторов БирманаШвингера. 
должны быть равномерно ограничены:

$$
\begin{gathered}
g_{e} \leqslant g_{0} \\
\operatorname{deg}(v)=\#\left\{v^{\prime} \in \mathscr{V}, v^{\prime} \sim v\right\} \leqslant \mathbf{d} .
\end{gathered}
$$

Теорема 3.2. Пусть граф $G$ удовлетворяет условиям (3.6), (3.7). Тогда для любой функиии $V \geqslant 0$

$$
n\left(s, \mathbf{B}_{V}\right) \geqslant(\mathbf{d}+1)^{-1} \nu\left(g_{0}(\mathbf{d}+1) s, V\right)
$$

u, следовательно, для любого $q>0$

$$
\left\|\mathbf{B}_{V}\right\|_{\Sigma_{q}} \geqslant c\|V\|_{\ell_{w}^{q}}, \quad\left\|\mathbf{B}_{V}\right\|_{\mathfrak{S}_{q}} \geqslant c\|V\|_{\ell^{q}}, \quad c=c\left(q, \mathbf{d}, g_{0}\right)>0 .
$$

Доказательство. Хорошо известно, что множество $\mathscr{V}$ может быть разбито на не более чем $\mathbf{d}+1$ непересекающихся подмножеств $\mathscr{V}_{j}$ так, что никакие две вершины из одного подмножества не смежны. Следовательно, для любого фиксированного $\tau>0$ множество $E(\tau, V)=\{v \in \mathscr{V}: V(v)>\tau\}$ разбивается в объединение не более чем $\mathbf{d}+1$ непересекающихся подмножеств $\Omega_{j}=E(\tau, V) \cap$ $\mathscr{V}_{j}$. Хотя бы для одного из них, скажем, $\Omega_{1}$, будет $\# \Omega_{1} \geqslant(\mathbf{d}+1)^{-1} \nu(\tau, V)$. Теперь рассмотрим подпространство $\mathscr{L} \subset \mathscr{H}(G)$, порожденное функциями $f_{v^{\prime}}(v)=$ $\delta_{v, v^{\prime}}, v^{\prime} \in \Omega_{1}$. Эти функции попарно ортогональны как в метрике (3.1), так и относительно квадратичной формы $\mathbf{b}_{V}$ в (3.4). Поэтому для любой функции $f(v)=\sum_{v^{\prime} \in \Omega_{1}} c_{v^{\prime}} \delta_{v, v^{\prime}} \in \mathscr{L}$ имеем

$$
\mathbf{a}_{G}[f]=\sum_{v \in \Omega_{1}}\left|c_{v}\right|^{2} \sum_{e \ni v} g_{e} \leqslant g_{0}(\mathbf{d}+1) \sum_{v \in \Omega_{1}}\left|c_{v}\right|^{2},
$$

в то время как $\mathbf{b}_{V}[f]=\sum_{v \in \Omega_{1}}\left|c_{v}\right|^{2} V(v) \geqslant \tau \sum_{v \in \Omega_{1}}\left|c_{v}\right|^{2}$. Таким образом, мы построили подпространство $\mathscr{L}, \operatorname{dim} \mathscr{L} \geqslant(\mathbf{d}+1)^{-1} \nu(\tau, V)$, на котором

$$
\mathbf{b}_{V}[f] \geqslant \tau g_{0}^{-1}(\mathbf{d}+1)^{-1} \mathbf{a}_{G}[f] .
$$

Применяя теперь вариационный принцип, получаем (3.8). Оценки (3.9) получаются из (3.8) стандартным образом.

\section{§4. Метрические графы: оценки сверху}

4.1. Лапласиан и разложение пространства. Каждое ребро е метрического графа $\Gamma$ рассматривается как прямолинейный отрезок длины $l_{e}>0$. Метрическому графу $\Gamma$ соответствует комбинаторный граф $G=G(\Gamma)$ с теми же множествами вершин $\mathscr{V}$ и ребер $\mathscr{E}$. Каждому ребру е графа $G(\Gamma)$ поставим в соответствие вес $g_{e}=l_{e}^{-1}$. Если $v$ - вершина графа $\Gamma$, то $\mathscr{S}(v)$ обозначает ее звезду: $\mathscr{S}(v)=\bigcup_{e \ni v} e$. Звезда $\mathscr{S}(e)$ ребра $e=\left(v, v^{\prime}\right)$ определяется как $\mathscr{S}(v) \cup \mathscr{S}\left(v^{\prime}\right)$.

Мера Лебега на ребрах индуцирует меру на $Г$, и наше базовое гильбертово пространство есть $L^{2}(\Gamma)$ по этой мере.

На пространстве $H^{1}(\Gamma)$ непрерывных функций $\varphi$ на $\Gamma$, таких, что $\varphi \in H^{1}(e)$ на каждом ребре и $\int_{\Gamma}\left(\left|\varphi^{\prime}\right|^{2}+|\varphi|^{2}\right) d y<\infty$, рассмотрим квадратичную форму

$$
\mathbf{a}_{\Gamma}[\varphi]:=\int_{\Gamma}\left|\varphi^{\prime}(y)\right|^{2} d y
$$


Лапласиан $\mathbf{A}_{\Gamma}$ в $L^{2}(\Gamma)$ определяется этой квадратичной формой. Он действует как $-d^{2} / d y^{2}$ на каждом ребре. Его область определения $\operatorname{Dom}\left(\mathbf{A}_{\Gamma}\right)$ состоит из функций $\varphi$, принадлежащих $H^{2}$ на каждом ребре, непрерывных в вершинах, удовлетворяющих условиям Кирхгофа и таких, что $\sum_{e \in \mathscr{E}}\|\varphi\|_{H^{2}(e)}^{2}<\infty$. Сначала изучим соотношения между показателями $\delta, D$ для полугрупп, порожденных операторами $\mathbf{A}_{\Gamma}$ в $L^{2}(\Gamma)$ и $\mathbf{A}_{G(\Gamma)}$ в $\ell^{2}(G(\Gamma))$.

С этой целью мы рассмотрим два предгильбертовых пространства, являющихся линейными подпространствами в пространстве $H_{\text {comp }}^{1}(\Gamma)$ функций из $H^{1}(\Gamma)$ с компактным носителем. Одно из них, $H_{\text {comp,pl }}^{1}(\Gamma)$, образовано кусочно линейными функциями, линейными на каждом ребре (отсюда обозначение $\mathrm{pl}$, piecewise linear). Каждая функция $\varphi \in H_{\text {comp,pl }}^{1}(\Gamma)$ однозначно определяется значениями $\varphi(v)$ в вершинах. Для последовательности $f=\{f(v)\}, v \in \mathscr{V}$, с конечным носителем обозначим через $J f$ единственную функцию из $H_{\text {comp,pl }}^{1}(\Gamma)$, такую, что $(J f)(v)=f(v)$ для любой вершины $v \in \mathscr{V}$. Отображение $J$ определяет изометрию между предгильбертовыми пространствами $H_{\text {comp,pl }}^{1}(\Gamma)$ с метрикой а эти предгильбертовы пространства посредством этой изометрии.

Другое подпространство - это пространство $H_{\mathrm{comp}, \mathscr{D}}^{1}$, состоящее из функций $\varphi \in H_{\text {comp }}^{1}(\Gamma)$, таких, что $\varphi(v)=0$ для всех $v \in \mathscr{V}$. Ясно, что

$$
H_{\mathrm{comp}}^{1}=H_{\mathrm{comp}, \mathrm{pl}}^{1} \oplus H_{\mathrm{comp}, \mathscr{D}}^{1}
$$

(ортогональная сумма относительно метрики $\mathbf{a}_{\Gamma}$ ). Обозначим через $\varphi_{\mathrm{pl}}$ и $\varphi_{\mathscr{D}}$ компоненты данной функции $\varphi$ относительно этого разложения.

4.2. Размерности метрического графа. Следующая теорема показывает, что локальная размерность лапласиана на $Г$ сколь угодно близка к 1 (что подтверждает интуитивное представление о локальной размерности), в то время как размерность на бесконечности такая же, как у $G$. Мы думаем, что в действительности оценка (4.3) справедлива с $\delta=1$. Тем не менее более слабый результат, который мы доказываем в теореме 4.1, п. 1, достаточен для главных выводов о спектральных оценках.

Теорема 4.1. 1. Для любого $\delta>1$

$$
M_{\mathbf{A}_{\Gamma}}(t) \leqslant C(\delta) t^{-\delta / 2}, \quad t \in(0,1) .
$$

2. Если длины ребер равномерно ограничены, т.е.

$$
l_{e} \leqslant l_{+} \quad \forall e \in \mathscr{E},
$$

u $M_{\mathbf{A}_{G}}(t)=O\left(t^{-D / 2}\right), D>2$, npu $t \rightarrow \infty$, mo $u M_{\mathbf{A}_{\Gamma}}(t)=O\left(t^{-D / 2}\right)$.

Доказательство. 1. Достаточно доказать $(4.3)$ для $\delta \in(1,2)$. Прежде всего, можно найти такое число $s>0$, что для любой точки $z$ в графе $\Gamma$ существует простой путь $S(z)$, содержащий $z$ и имеющий длину $s$. Чтобы показать это, фиксируем какую-либо вершину $v_{0} \in \mathscr{V}$ и положим $s=\min \left\{l_{e}: e \in \mathscr{S}\left(v_{0}\right)\right\}$. Тогда для $z \in \mathscr{S}\left(v_{0}\right)$ выбор пути очевиден. Если же $z \notin \mathscr{S}\left(v_{0}\right)$, то имеется простой путь, соединяющий $z$ и $v_{0}$, и длина его больше $s$. В качестве $S(z)$ можно тогда принять отрезок длины $s$ этого пути, содержащий $z$. В дальнейшем мы рассматриваем этот путь $S(z)$ как интервал. 
Для данной точки $z \in \Gamma$ рассмотрим оператор $T_{z}$ сужения функций, заданных на $\Gamma$, на $S(z)$. Он, очевидно, ограничен как оператор из $H^{1}(\Gamma)=\operatorname{Dom}\left(\mathbf{A}_{\Gamma}^{1 / 2}\right)$ в $H^{1}(S(z))$ и из $L^{2}(\Gamma)$ в $L^{2}(S(z))$, и в обоих случаях его норма не превосходит 1. Интерполируя, получаем, что $T_{z}$ ограничен и имеет норму не больше 1 как опеpaтор из $\operatorname{Dom}\left(\mathbf{A}_{\Gamma}^{\delta / 4}\right)$ в $H^{\delta / 2}(S(z))$ для любого $\delta \in(0,2)$. При $\delta>1$ пространство $H^{\delta / 2}(S(z))$ вложено в $C(S(z))$, при этом норма оператора вложения не зависит от $z$. Пользуясь произвольностью $z$, заключаем, что $\left(\mathbf{I}+\mathbf{A}_{\Gamma}\right)^{-\delta / 4}$ ограничен как оператор из $L^{2}(\Gamma)$ в $L^{\infty}(\Gamma)$. Далее,

$$
\exp \left(-t \mathbf{A}_{\Gamma}\right)=t^{-\delta / 4}\left(\mathbf{I}+\mathbf{A}_{\Gamma}\right)^{-\delta / 4}\left[\left(t\left(\mathbf{I}+\mathbf{A}_{\Gamma}\right)\right)^{\delta / 4} \exp \left(-t \mathbf{A}_{\Gamma}\right)\right]
$$

По спектральной теореме оператор в квадратных скобках ограничен в $L^{2}(\Gamma)$ равномерно по $t \in(0,1)$, откуда следует, что

$$
\left\|\exp \left(-t \mathbf{A}_{\Gamma}\right)\right\|_{L^{2}(\Gamma) \rightarrow L^{\infty}(\Gamma)}=O\left(t^{-\delta / 4}\right), \quad t \in(0,1) .
$$

Эта оценка и двойственная ей влекут за собой (4.3); детали и дальнейшие ссылки см. в [13, разд. 2.1].

2. По теореме II.3.1 из [17] достаточно доказать неравенство Соболева

$$
\|\varphi\|_{L^{p}}^{2} \leqslant C(p) \mathbf{a}_{\Gamma}[\varphi], \quad p=p(D)=2 D /(D-2),
$$

для произвольной функции $\varphi \in H_{\text {comp }}^{1}(\Gamma)$. Поскольку разложение (4.2) ортогонально, достаточно установить (4.5) отдельно для компонент $\varphi_{\mathrm{pl}}$ и $\varphi_{\mathscr{D}}$. Норма в $L^{p}(\Gamma)$ функции $\varphi_{\mathrm{pl}}=J f$ оценивается сверху через норму функции $f$ в $\ell^{p}(G)$, так что неравенство Соболева для $\varphi_{\mathrm{pl}}$ следует из соответствующего неравенства для $f$. Для $\varphi_{\mathscr{D}}$ соответствующее неравенство на каждом ребре выполняется в силу (4.4) с общей константой. Суммируя, получаем (4.5).

Таким образом, общие результаты $\S 2$ применимы к случаю метрических графов. Однако приводимый ниже анализ дает более полную и детальную картину.

4.3. Операторы Бирмана-Швингера. Как всегда, мы предполагаем, что комбинаторный граф $G$ удовлетворяет (3.2). Следовательно, в силу теоремы 4.1 и оценки (2.3) пространство $\mathscr{H}^{1}=\mathscr{H}^{1}(\Gamma)$, определенное как пополнение пространства $H_{\text {comp }}^{1}(\Gamma)$ по метрике а, является функциональным гильбертовым пространством, вложенным в $L^{p}(\Gamma)$.

Замыкая оба слагаемых в правой части (4.2) по той же метрике, мы получим гильбертовы пространства $\mathscr{H}_{\mathrm{pl}}^{1}$ и $\mathscr{H}_{\mathscr{D}}^{1}$, так что $\mathscr{H}_{\mathscr{D}}^{1}(\Gamma)=\sum_{e \in \mathscr{E}}^{\oplus} H^{1,0}(e)$ и

$$
\mathscr{H}^{1}(\Gamma)=\mathscr{H}_{\mathrm{pl}}^{1} \oplus \mathscr{H}_{\mathscr{D}}^{1} .
$$

Изометрия $J$ расширяется до изометрии пространств $\mathscr{H}(G)$ и $\mathscr{H}_{\mathrm{pl}}^{1}$. Квадратичная форма (2.5) в нашем случае равна

$$
\mathbf{b}_{V}[\varphi]=\int_{\Gamma} V(y)|\varphi(y)|^{2} d y .
$$

В общей ситуации разложение (4.6) не диагонализует соответствующий оператор $\mathbf{B}_{V}$. Тем не менее полезно рассмотреть операторы $\mathbf{B}_{V, \text { pl }}$ и $\mathbf{B}_{V, \mathscr{D}}$, действующие в $\mathscr{H}_{\mathrm{pl}}^{1}$ и $\mathscr{H}_{\mathscr{D}}^{1}$ соответственно и порожденные квадратичной формой (4.7), суженной на соответствующие подпространства. Спектральные оценки для $\mathbf{B}_{V}$ легко выводятся из оценок для этих операторов. Действительно, бла- 
годаря неравенству $\mathbf{b}_{V}[\varphi] \leqslant 2\left(\mathbf{b}_{V}\left[\varphi_{\mathrm{pl}}\right]+\mathbf{b}_{V}\left[\varphi_{\mathscr{D}}\right]\right)$, ясно, что оператор $\mathbf{B}_{V}$ будет ограниченным (или компактным), если и только если таковы оба этих оператора. Более того, мы имеем (в случае компактности)

$$
\max \left\{n\left(s, \mathbf{B}_{V, \mathrm{pl}}\right), n\left(s, \mathbf{B}_{V, \mathscr{D}}\right)\right\} \leqslant n\left(s, \mathbf{B}_{V}\right) \leqslant n\left(s / 2, \mathbf{B}_{V, \mathrm{pl}}\right)+n\left(s / 2, \mathbf{B}_{V, \mathscr{D}}\right) .
$$

Теперь мы готовы перейти к спектральным оценкам сверху для операторов $\mathbf{B}_{V, D}$ и $\mathbf{B}_{V, \mathrm{pl}}$. Они приведены в леммах 4.2 и 4.3. Окончательные оценки для исходного оператора $\mathbf{B}_{V}$ сформулированы в следующем параграфе. Там же установлены и оценки снизу, показывающие точность результата.

Структура операторов $\mathbf{B}_{V, \mathscr{D}}$ проста:

$$
\mathbf{B}_{V, \mathscr{D}}=\sum_{e \in \mathscr{E}}^{\oplus} \mathbf{B}_{V, e, \mathscr{D}},
$$

где $\mathbf{B}_{V, e, \mathscr{D}}$ обозначает оператор в $H^{1,0}(e)$, порожденный квадратичной формой типа (4.7) с интегрированием по ребру $e$.

Рассмотрим квадратичную форму (4.7) для $\varphi \in \mathscr{H}_{\mathrm{pl}}^{1}(\Gamma)$. Пусть $f=\{f(v)\}-$ сужение функции $\varphi$ на $\mathscr{V}$, т. е. $f(v)=\varphi(v)$ для любой вершины $v \in \mathscr{V}$. Тогда

$$
\mathbf{b}_{V}\left[\varphi_{\mathrm{pl}}\right]=\mathbf{b}_{V}[J f]=\sum_{e \in \mathscr{E}} \int_{e} V(y)|(J f)(y)|^{2} d y .
$$

Соответствующий оператор на $\mathscr{H}_{\mathrm{pl}}^{1}$ есть $\mathbf{B}_{V, \mathrm{pl}}$. Рассмотрим также оператор $\widehat{\mathbf{B}}_{V, \mathrm{pl}}$ в $\mathscr{H}^{1}(G)$, порожденный квадратичной формой $\widehat{\mathbf{b}}_{V}[f] \equiv \mathbf{b}_{V}[J f]$. Ясно, что операторы $\mathbf{B}_{V, \mathrm{pl}}$ и $\widehat{\mathbf{B}}_{V, \mathrm{pl}}$ унитарно эквивалентны.

4.4. Оператор $\mathbf{B}_{\boldsymbol{V}, \mathscr{D}}$. Ортогональное разложение (4.9) сводит изучение спектра оператора $\mathbf{B}_{V, \mathscr{D}}$ к той же задаче для семейства конечных интервалов, что делает его элементарным.

Мы ставим в соответствие потенциалу $V$ последовательность

$$
\boldsymbol{\eta}_{V}=\left\{\eta_{V}(e)\right\}, \quad \eta_{V}(e)=l_{e} \int_{e} V d y, \quad e \in \mathscr{E} .
$$

Хорошо известно (см., например, оценку (4.8) и теорему 4.6 в [3], где следует положить $l=m=1$ ), что

$$
\begin{array}{cc}
n\left(\lambda, \mathbf{B}_{V, e, \mathscr{D}}\right) \leqslant C \lambda^{-1 / 2} \sqrt{\eta_{V}(e)} & \forall \lambda>0, \\
\lambda^{1 / 2} n\left(\lambda, \mathbf{B}_{V, e, \mathscr{D}}\right) \rightarrow \frac{1}{\pi} \int_{e} \sqrt{V} d x, \quad \lambda \rightarrow 0 .
\end{array}
$$

Пусть $\nu\left(s, \boldsymbol{\eta}_{V}\right)=\#\left\{e: \eta_{V}(e)>s\right\}, s>0,-$ функция распределения последовательности (4.11). Будем говорить, что $\boldsymbol{\eta}_{V} \rightarrow 0$, если $\nu\left(s, \boldsymbol{\eta}_{V}\right)<\infty$ для любого $s>0$.

Следующее утверждение выводится из (4.9) с помощью (4.12) и (4.13).

Лемма 4.2. 1. Если $\boldsymbol{\eta}_{V} \in \ell^{\infty}$, то оператор $\mathbf{B}_{V, \mathscr{D}}$ ограничен и

$$
\left\|\mathbf{B}_{V, \mathscr{D}}\right\| \leqslant C\left\|\boldsymbol{\eta}_{V}\right\|_{\ell^{\infty}}, \quad C>0 .
$$

Eсли $\boldsymbol{\eta}_{V} \rightarrow 0$, то оператор $\mathbf{B}_{V, \mathscr{D}}$ компактен.

2. Для любого $q \in(1 / 2, \infty)$

$$
\left\|\mathbf{B}_{V, \mathscr{D}}\right\|_{\mathfrak{S}_{q}} \leqslant C(q)\left\|\boldsymbol{\eta}_{V}\right\|_{\ell^{q}}, \quad\left\|\mathbf{B}_{V, \mathscr{D}}\right\|_{\Sigma_{q}} \leqslant C(q)\left\|\boldsymbol{\eta}_{V}\right\|_{\ell_{w}^{q}} .
$$


3. Пусть $\boldsymbol{\eta}_{V} \in \ell^{1 / 2}$. Тогда

$$
\left\|\mathbf{B}_{V, \mathscr{D}}\right\|_{\Sigma_{1 / 2}} \leqslant C \sum_{e \in \mathscr{E}} \sqrt{\eta_{V}(e)} \quad u \quad \lambda^{1 / 2} n\left(\lambda, \mathbf{B}_{V, \mathscr{D}}\right) \rightarrow \frac{1}{\pi} \int_{\Gamma} \sqrt{V} d y, \quad \lambda \rightarrow 0 .
$$

Доказательство. Обоснование довольно стандартно, см., например, [12], и мы докажем только утверждение 2 для классов $\Sigma_{q}$. Если $\boldsymbol{\eta}_{V} \in \ell_{w}^{q}$, то после подходящей перенумерации ребер $e_{j}$ имеем $\eta_{V}\left(e_{j}\right) \leqslant M j^{-1 / q}$. Следовательно, в силу (4.12)

$$
n\left(\lambda, B_{V, e_{j}, \mathscr{D}}\right) \leqslant C M^{1 / 2} \lambda^{-1 / 2} j^{-1 / 2 q} .
$$

В частности, $n\left(\lambda, B_{V, e_{j}, \mathscr{D}}\right)=0$, если $j>C^{2 q} M^{q} \lambda^{-q}$. Поэтому

$$
n\left(\lambda, B_{V, \mathscr{D}}\right)=\sum_{e} n\left(\lambda, B_{V, e, \mathscr{D}}\right) \leqslant C M^{1 / 2} \lambda^{-1 / 2} \sum_{j \leqslant C^{2 q} M^{q} \lambda^{-q}} j^{-1 / 2 q},
$$

и так как $2 q>1$, то $n\left(\lambda, B_{V, \mathscr{D}}\right) \leqslant C^{\prime} M^{q} \lambda^{-q}$, откуда получаем требуемое.

4.5. Оператор $\mathbf{B}_{V, \mathbf{p l}}$. Сравним наш оператор $\mathbf{B}_{V, \mathrm{pl}}$ (или, что равносильно, $\left.\widehat{\mathbf{B}}_{V, \mathrm{pl}}\right)$ с оператором $\mathbf{B}_{\varkappa_{V}}$, где дискретный потенциал $\varkappa_{V}=\left\{\varkappa_{V}(v)\right\}$ выбирается специальным образом:

$$
\varkappa_{V}(v)=\int_{\mathscr{S}(v)} V d y=\sum_{e \ni v} l_{e}^{-1} \eta_{V}(e) \quad \forall v \in \mathscr{V} .
$$

Вернемся к квадратичной форме в (4.10). Выберем ребро $e=\left(v, v^{\prime}\right)$. Отождествляя $е$ с интервалом $\left(0, l_{e}\right)$, получаем для $f \in \mathscr{H}(G)$

$$
\begin{aligned}
\int_{e} V(y)|(J f)(y)|^{2} d y & =l_{e}^{-2} \int_{0}^{l_{e}} V(y)\left|f(v)\left(l_{e}-y\right)+f\left(v^{\prime}\right) y\right|^{2} d y \\
& \leqslant \max \left\{|f(v)|^{2},\left|f\left(v^{\prime}\right)\right|^{2}\right\} \int_{e} V(y) d y .
\end{aligned}
$$

Суммируя интегралы по всем ребрам $e \in \mathscr{E}$, имеем

$$
\widehat{\mathbf{b}}_{V}[f] \leqslant \mathbf{b}_{\varkappa_{V}}[f] .
$$

Отсюда вытекает следующий результат.

Лемма 4.3. Предположим, что оператор $\mathbf{B}_{\varkappa_{V}}$ на комбинаторном графе $G(\Gamma)$ ограничен, компактен или лежит в одном из классов $\mathfrak{S}_{q}, \Sigma_{q}, q>0$. Тогда то же верно для оператора $\mathbf{B}_{V, \mathrm{pl}}$ и оченка

$$
\left\|\mathbf{B}_{V, \mathrm{pl}}\right\| \leqslant\left\|\mathbf{B}_{\varkappa_{V}}\right\|
$$

справедлива в (квази)норме соответствующего класса.

\section{§5. Метрические графы: оценки в классах Шаттена и асимптотика спектра}

5.1. Оценки снизу. Начнем с простой леммы из теории графов.

Лемма 5.1. Пусть граф $G(\Gamma)$ удовлетворяет условию (3.7). Тогда множество ребер $\mathscr{E}$ можно раскрасить не более чем в $2 \mathbf{d}^{2}+1$ иветов (разбить не более чем на $2 \mathbf{d}^{2}+1$ подмножеств $\left.\mathscr{E}_{j}\right)$ так, что $\mathscr{S}(e) \cap \mathscr{S}\left(e^{\prime}\right)=\varnothing$ для любых ребер $е \neq e^{\prime}$ одного ивета. 
Доказательство. Упорядочим ребра произвольным образом и будем красить по очереди. Для каждого очередного ребра $e$ имеется не более $2 \mathbf{d}^{2}$ уже окрашенных ребер $e^{\prime}$, для которых $\mathscr{S}(e) \cap \mathscr{S}\left(e^{\prime}\right) \neq \varnothing$, так что ребро е можно окрасить в неиспользованный цвет.

Теперь мы в состоянии вывести оценки снизу для нашего исходного оператора $\mathbf{B}_{V}$. Как и теорема 3.2 , они требуют дополнительных ограничений: а именно мы предполагаем, что длины ребер $l_{e}$ ограничены и отделены от нуля:

$$
0<l_{-} \leqslant l_{e} \leqslant l_{+} \quad \forall e \in \mathscr{E},
$$

а также что выполняется условие (3.7). Заметим, что из левого неравенства в (5.1) следует (3.6) для комбинаторного графа $G(\Gamma)$.

Лемма 5.2. Пусть выполнены условия (3.7) и (5.1). Тогда

(1) если оператор $\mathbf{B}_{V}$ ограничен, то $\boldsymbol{\eta}_{V} \in \ell_{\infty} u\left\|\mathbf{B}_{V}\right\| \geqslant c\left\|\boldsymbol{\eta}_{V}\right\|_{\infty}$;

(2) если оператор $\mathbf{B}_{V}$ компактен, то последовательность $\boldsymbol{\eta}_{V}$ сходится $\kappa$ нулю; более того, существуют константы $c^{\prime}, c^{\prime \prime}$, зависящие только от $\mathbf{d} u$ $l_{+} / l_{-}$, такие, что

$$
n\left(s, \mathbf{B}_{V}\right) \geqslant c^{\prime} \nu\left(c^{\prime \prime} s, \boldsymbol{\eta}_{V}\right) \quad \forall s>0 .
$$

Доказательство. Приводимая схема рассуждений многократно использовалась в литературе, см., например, [5], [12], [15]. Пусть $e=\left(v, v^{\prime}\right)-$ ребро. Рассмотрим функцию $\varphi_{e} \in \mathscr{H}_{p l}^{1}(\Gamma)$, такую, что $\varphi_{e}(y)=1$ для $y \in e$ и $\varphi_{e}(y)=0$ вне множества $\mathscr{S}(e)=\mathscr{S}(v) \cup \mathscr{S}\left(v^{\prime}\right)$. Такая функция единственна и $\int_{\Gamma}\left|\varphi_{e}^{\prime}\right|^{2} d y \leqslant 2(\mathbf{d}-1) l_{-}^{-1}$. Более того, $\int_{\Gamma} V\left|\varphi_{e}\right|^{2} d y \geqslant l_{+}^{-1} \eta_{V}(e)$ и, следовательно,

$$
\frac{\int_{\Gamma} V\left|\varphi_{e}\right|^{2} d y}{\int_{\Gamma}\left|\varphi_{e}^{\prime}\right|^{2} d x} \geqslant(2 \mathbf{d}-2)^{-1} l_{-} l_{+}^{-1} \eta_{V}(e) .
$$

Отсюда непосредственно вытекает утверждение (1).

Далее, если для ребер $e_{1}, e_{2}$ множества $\mathscr{S}\left(e_{1}\right), \mathscr{S}\left(e_{2}\right)$ не пересекаются, то соответствующие функции $\varphi_{e_{1}}, \varphi_{e_{2}}$ ортогональны в $\mathscr{H}^{1}(\Gamma)$ и в пространстве $L^{2}$ с весом $V$. Будем называть подмножество $F \subset \mathscr{E}$ разделенным, если множества $\mathscr{S}(e), e \in F$, попарно не пересекаются. Сужая квадратичную форму $\mathbf{b}_{V}$ на линейную оболочку функций $\varphi_{e}, e \in F$, мы получаем оператор, собственные значения которого, с точностью до порядка нумерации, суть в точности числа в левой части формулы (5.3). Следовательно, числа $\eta_{V}(e)$, упорядоченные по убыванию, не превосходят собственных значений $\lambda_{n}\left(\mathbf{B}_{V}\right)$ (или даже $\lambda_{n}\left(\mathbf{B}_{V, \mathrm{pl}}\right)$ ), умноженных на $2(\mathbf{d}-1) l_{+} l_{-}^{-1}$. По лемме 5.1 множество $\mathscr{E}$ разбивается на не более чем $2 \mathbf{d}^{2}+1$ разделенных подмножеств, откуда получаем утверждение (2).

Заметим, что подобная нижняя оценка для оператора $\mathbf{B}_{V, \mathscr{D}}\left(\right.$ вместо $\mathbf{B}_{V}$ ) не имеет места.

5.2. Оператор $\mathbf{B}_{V}$. Теперь несложно получить следующий результат для исходного оператора $\mathbf{B}_{V}$ в пространстве $\mathscr{H}^{1}(\Gamma)$, порожденного квадратичной формой (4.7). Мы сравним его с «дискретным» оператором $\mathbf{B}_{\varkappa_{V}}$ в пространстве $\mathscr{H}(G), G=G(\Gamma)$, где дискретный потенциал $\varkappa_{V}$ определен формулой (4.16).

Теорема 5.3. Пусть Г - метрический граф, для которого справедливо неравенство (4.4) и $D>2$. Тогда оператор $\mathbf{B}_{V}$ ограничен (соответственно компактен, лежит в одном из классов $\mathfrak{S}_{q}, \mathfrak{S}_{q, w}$ с $\left.q>1 / 2\right)$, если (а при предположениях (3.7) и (5.1) и только если) выполняются следующие два условия: 
(1) оператор $\mathbf{B}_{\varkappa_{V}}$ лежит в соответствующем классе;

(2) последовательность $\varkappa_{V}$ лежит в $\ell^{\infty}$ (соответственно в $c_{0}, \ell^{q}$ или $\ell_{w}^{q}$ ).

Если $q<D / 2$, то условие (1) следует из (2) и, значит, его можно и не налагать.

Заметим, что последнее утверждение следует из верхних оценок для комбинаторных графов, см. п. 2 теоремы 3.1.

Замечание 5.4. В частности, $\mathbf{B}_{V} \in \mathfrak{S}_{1}$, если (а при предположениях (5.1) и (3.7) и только если) $\int_{\Gamma} V d y<\infty$.

Доказательство теоремы 5.3. Утверждение «если» получается из лемм 4.2 и 4.3 с помощью второго неравенства из (4.8).

Утверждение «только если»: если $\mathbf{B}_{V}$ обладает одним из свойств из нашего предположения, то этим же свойством обладает оператор $\mathbf{B}_{\varkappa_{V}}$ благодаря первому неравенству в (4.8). Условие (2) выполнено по лемме 5.2.

Последний результат показывает, что спектральные свойства оператора $\mathbf{B}_{V}$ в значительной степени определяются теми же свойствами его дискретного аналога. Отметим, что результат для $q>D / 2$ следует рассматривать как «условный»: в самом деле, наши результаты для общих комбинаторных графов (теорема 3.1 ) относятся только к случаю $q \leqslant D / 2$. Для дальнейших результатов необходима дополнительная информация о структуре графа $G$. В важном случае $G=\mathbb{Z}^{d}, d \geqslant 3$, такие результаты получены в [15].

В частности, там предложена конструкция «разреженных потенциалов», позволяющая строить дискретный потенциал, для которого оператор $\mathbf{B}_{V}$ имеет произвольную предписанную асимптотику спектра. Отметим, что такая конструкция обобщается на произвольные комбинаторные графы с $D>2$. Этот материал будет изложен отдельно.

Теорема 5.3 не охватывает пограничного случая $q=1 / 2$. Если $q=1 / 2$, то можно привести простое достаточное условие для $\mathbf{B}_{V} \in \mathfrak{S}_{1 / 2, w}$.

Теорема 5.5. Пусть $\boldsymbol{\eta}_{V} \in \ell^{1 / 2}$. Тогда

$$
\left\|\mathbf{B}_{V}\right\|_{\Sigma_{1 / 2}} \leqslant C\left\|\boldsymbol{\eta}_{V}\right\|_{\ell^{1 / 2}}
$$

или, что то же самое,

$$
N_{-}(\mathbf{A}-\alpha V) \leqslant C \alpha^{1 / 2} \sum_{e \in \mathscr{E}} \boldsymbol{\eta}_{V}(e)^{1 / 2} .
$$

Имеет место вейлевская асимптотика

$$
N_{-}(\mathbf{A}-\alpha V) \sim \frac{\alpha^{1 / 2}}{\pi} \int_{\Gamma} \sqrt{V} d y .
$$

Доказательство. По лемме 4.2 оператор $\mathbf{B}_{V, \mathscr{D}}$ удовлетворяет оценке (5.4) и асимптотике (5.5). Поскольку $\varkappa_{V}$ лежит в $\ell^{1 / 2}$ одновременно с $\boldsymbol{\eta}_{V}$, оператор $\mathbf{B}_{V, \mathrm{pl}}$ лежит в $\mathfrak{S}_{1 / 2}$, а следовательно, и в $\Sigma_{1 / 2}^{(0)}$, с соответствующей оценкой для квазинормы. Поэтому он не влияет на спектральную асимптотику порядка $1 / 2$. Отсюда следуют оба утверждения теоремы.

Необходимое и достаточное условие справедливости формул (5.4) и (5.5) может быть получено по аналогии с [11]. Мы его здесь не приводим. 


\section{ЛитеРАТУРА}

[1] Й. Берг, Й. Лефстрем, Интерполяционнъе пространства. Введение, Мир, М., 1980 .

[2] М. Ш. Бирман, О спектре сингулярных граничных задач, Матем. сб., 55:2 (1961), $125-174$.

[3] М. Ш. Бирман, М. З. Соломяк, Количественный анализ в теоремах вложения Соболева и приложения $\kappa$ спектралъной теории, в кн.: Десятая математическая школа, Институт математики АН УССР, Киев, 1974.

[4] M. Sh. Birman, M. Z. Solomyak, Spectral theory of selfadjoint operators in Hilbert space, D. Reidel Publishing Co., Dordrecht, 1987.

[5] M. Sh. Birman, M. Z. Solomyak, Estimates for the number of negative eigenvalues of the Schrödinger operator and its generalizations, in: Adv. Soviet Math., vol. 7, Amer. Math. Soc., Providence, RI, 1991, 1-55.

[6] T. Coulhon, A. Grigoryan, Random walks on graphs with regular volume growth, Geom. Funct. Anal., 8:4 (1998), 656-701.

[7] И. Ц. Гохберг, М. Г. Крейн, Введение в теорию линейных несамосопряженных операторов, Наука, М., 1965.

[8] A. Grigoryan, J. Hu, Off-diagonal upper estimates for the heat kernel of the Dirichlet forms on metric spaces, Invent. Math., 174:1 (2008), 81-126.

[9] Ф. Харари, Теория графов, Мир, М., 1973; УРСС, М., 2003.

[10] S. Molchanov, B. Vainberg, On general Cwikel-Lieb-Rozenblum and Lieb-Thirring inequalities, in: Around the Research of Vladimir Maz'ya III, Int. Math. Ser., vol. 13, Springer-Verlag, New York, 2010, 201-246.

[11] K. Naimark, M. Solomyak, Regular and pathological eigenvalue behaviour for the equation $-\lambda u^{\prime \prime}=V u$ on the semiaxis, J. of Functional Analysis, 151:2 (1997), 504-530.

[12] K. Naimark, M. Solomyak, Eigenvalue estimates for the weighted Laplacian on metric trees, Proc. London Math. Soc., 80:3 (2000), 690-724.

[13] Г. Розенблюм, М. Соломяк, Оценка ЦЛР для генераторов положительных полугрупп и полугрупп, доминируемых положительными, Алгебра и анализ, 9:6 (1997), 214-236.

[14] G. Rozenblum, M. Solomyak, Counting Schrödinger boundstates: semiclassics and beyond, in: Sobolev Spaces in Mathematics. II. Applications in Analysis and Partial Differential Equations, Int. Math. Ser., vol. 9, Springer-Verlag, New York, 2009, 329354.

[15] Г. Розенблюм, М. Соломяк, Об оценках спектра для оператора Шрёдингера на $\mathbb{Z}^{d}, d \geqslant 3$, Пробл. матем. анализа, 2009, № 41, 107-126.

[16] L. Saloff-Coste, Sobolev inequalities in familiar and unfamiliar settings, in: Sobolev Spaces in Mathematics. I, Int. Math. Ser., vol. 8, Springer-Verlag, New York, 2009, 299-343.

[17] N. Th. Varopoulos, L. Saloff-Coste, T. Coulhon, Analysis and Geometry on Groups, Cambridge University Press, Cambridge, 1992.

Department of Mathematics, Chalmers University of Technology and The University of Gothenburg e-mail: grigori@chalmers.se

Department of Mathematics, Weizmann Institute, Rehovot, Israel e-mail: solom@wisdom.weizmann.ac.il 